\title{
Avaliação da inflamabilidade de espécies nativas do cerrado
}

\author{
Marcileia Dias de Oliveira * \\ Josué Luiz Marinho Junior \\ Francisca de Cássia Silva da Silva \\ Ângela Mendes Stival \\ Bárbara Gomes Ferreira \\ Marcos Giongo \\ Universidade Federal do Tocantins \\ Rua Delfino Aguiar 737, Quadra 2, Lote 3, CEP 77.405-040, Gurupi - TO, Brasil \\ * Autor para correspondência \\ oliveira.mdias@gmail.com
}

Submetido em 28/05/2020

Aceito para publicação em 06/10/2020

\section{Resumo}

$\mathrm{Na}$ ocorrência de incêndios florestais, a propagação se deve principalmente às características das folhas. Elas funcionam como potencializador ou retardador na propagação do fogo, visto que folhas com baixa inflamabilidade servem como barreiras de proteção contra incêndios. O presente trabalho teve por finalidade analisar a inflamabilidade de seis espécies do cerrado para potencial uso em cortinas de segurança. Para a realização do experimento, foi utilizado o epirradiador com uma faixa de temperatura controlada em torno de $600^{\circ} \mathrm{C}$. Das espécies avaliadas, foram coletadas 50 amostras de $1 \mathrm{~g}( \pm 0,1 \mathrm{~g})$ de cada espécie. Foram realizadas 50 repetições de queima, na qual se pôde analisar o Tempo para Ignição (TI), Frequência de Ignição (FI), Duração de Combustão (DC), Índice de Combustão (IC) e Valor de Inflamabilidade (VI). Os resultados obtidos dos VI das espécies foram: Protium heptaphyllum igual a 1 (fracamente inflamável); Caryocar brasiliense Cambess., Byrsonima verbascifolia (L.) DC., Acacia polyphylla e Terminalia argentea Mart. iguais a 3 (inflamáveis); e a espécie Lafoensia pacari igual a 4 (altamente inflamável). Deste modo, conclui-se que a espécie P. heptaphyllum apresenta potencial para uso em cortina de segurança na prevenção de incêndios florestais.

Palavras-chave: Epirradiador; Incêndios Florestais; Lafoensia pacari; Protium heptaphyllum

\section{Abstract}

Flammability assessment of native species of the cerrado. In the event of forest fires, the spread is mainly due to the characteristics of the leaves. In a way, the leaves act as a potentiator or retarder for the spread of fires, since the ones with low flammability can serve as fire protection barriers. The purpose of this work was to analyze the flammability of six species of the cerrado for potential use in fuelbreak. To carry out the experiment, an epirator with a controlled temperature range of around $600^{\circ} \mathrm{C}$ was used. From the evaluated species, 50 samples of $1 \mathrm{~g} \mathrm{(} \pm 0.1 \mathrm{~g})$ of each species were collected. 50 firing repetitions were performed, in which it was possible to analyze the Ignition Time (TI), Ignition Frequency (FI), Combustion Duration (DC), Combustion 
Index (CI) and Flammability Value (VI). The results obtained from the species flammability values were: Protium heptaphyllum equal to 1 (weakly flammable); Caryocar brasiliense Cambess., Byrsonima verbascifolia (L.) DC., Acacia polyphylla and Terminalia argentea Mart. equal to 3 (flammable); and the species Lafoensia pacari equal to 4 (highly flammable). Thus, it is concluded that the species $P$. heptaphyllum has potential for use as a fuelbreak to prevent forest fires.

Key words: Epirradiador; Forest fires; Lafoensia pacari; Protium heptaphyllum

\section{Introdução}

O fogo é um fenômeno físico-químico da combinação do oxigênio com um combustível, levando a produção de calor, luz e chama (SOARES; BATISTA, 2017). Nos combustíveis florestais, essa combustão passa pelo pré-aquecimento (com a secagem, aquecimento e destilação), depois segue-se a combustão dos gases (os componentes voláteis decompostos incendeiam) e, por fim, tem-se a incandescência (combustão do carbono com intensa liberação de calor) (SIMPSON et al., 2015).

A ocorrência de incêndios florestais é um dos principais fatores que ameaçam a biodiversidade do cerrado, emitindo $\mathrm{CO}_{2}$ na atmosfera, contribuindo para o aquecimento do planeta bem como para alterações da paisagem, além de acarretar prejuízos econômicos para a população (SILVEIRA et al., 2019). Em todo o mundo, preocupações sobre o impacto nas florestas, causados pelos incêndios, estão cada vez mais focados na paisagem interface florestal-urbana (WUI), onde sua ocorrência é alta, podendo afetar pessoas e estruturas (ETLINGER; BEALL, 2004).

As causas e frequências dos incêndios florestais estão relacionados à meteorologia, e a propagação é influenciada pela topografia, pelo material combustível e pelo teor de umidade das espécies (KOVALSYKI et al., 2019). Vegetações savânicas apresentam condições que facilitam a ocorrência de incêndios (clima seco, altas temperaturas e fortes ventos) (BATISTA et al., 2018). O Cerrado brasileiro, devido à sua vegetação intrínseca, é adaptado à ação do fogo que ocorre de maneira natural (MCLAUCHLAN et al., 2020).

De acordo com Hernando (2009), a inflamabilidade pode ser compreendida como sendo a facilidade com que algo queima ou entra em ignição, causando fogo e/ou combustão. Para minimizar a ocorrência de incêndios florestais, análises de inflamabilidade das espécies são realizadas com frequência, como forma de identificação de espécies com baixa inflamabilidade para a adoção de medidas de prevenção contra incêndios. Na região do Mediterrâneo, por exemplo, onde há uma alta incidência de incêndios florestais, diversos autores realizaram análises de inflamabilidade de variadas espécies florestais na região (HACHMI et al., 2011; GANTEAUME et al., 2011; MOLINA et al., 2017; FRÉJAVILLE et al., 2018). Uma das medidas de prevenção de combate a incêndios é a elaboração de barreiras verdes, também chamadas de cortinas de segurança.

Batista et al. (2012) esclarecem que o objetivo da barreira verde é retardar a propagação e a intensidade do fogo, evitando que o fogo se espalhe pelas copas das árvores e contribuindo para o controle e combate aos incêndios. Para garantir a eficiência dessas barreiras, Essaghi et al. (2017) destacam a importância do teste de chama, utilizado para se avaliar o nível de dificuldade necessária para que ocorra a combustão.

Anderson (1970) concluiu que, ao combinar a ignição, a sustentabilidade e a combustilidade, torna-se possível a avaliação do potencial de fogo de combustíveis florestais.

O objetivo deste trabalho foi avaliar o potencial de inflamabilidade de algumas espécies do Cerrado, para potencial uso em cortinas de segurança.

\section{Material e Métodos}

Para a avaliação da inflamabilidade de combustível, foi utilizado o protocolo proposto por Valette (1992) e Petriccione (2006).

Foram selecionadas folhas vivas, de mesma idade, de seis espécies arbóreas, extraídas na Universidade Federal de Tocantins, campus Gurupi - TO, sendo elas: Lafoensia pacari (Dedaleiro), Terminalia argentea Mart. 
(Capitão-do-Campo), Acacia polyphylla (Monjoleiro), Byrsonima verbascifolia (L.) DC. (Murici do cerrado), Caryocar brasiliense Cambess. (Pequi) e Protium heptaphyllum (Amescla). O critério estabelecido para a escolha deveu-se ao fato de as espécies serem umas das mais recorrentes em áreas do cerrado.

As espécies foram amostradas em outubro de 2018, durante o período considerado úmido no Tocantins, que se estende de outubro a abril. De acordo com Nelson (2001), o teor de umidade do combustível desempenha um papel crítico na ignição, propagação e consumo de combustível de um incêndio florestal. Além do que, dependendo do teor de umidade e do fluxo de calor de um incêndio, a vegetação pode atuar como fonte de calor ou dissipador de calor, contribuindo para disparar energia ou retardar a propagação e a intensidade do fogo.

Para identificar o teor de umidade, foi utilizada a equação 1, utilizada por Batista (1990), na qual duas subamostras de $80 \mathrm{~g}$ de cada espécie foram pesadas em balança de precisão e posteriormente colocadas em estufa a $75^{\circ} \mathrm{C}$, por $48 \mathrm{~h}$, de forma que foram obtidas a massa úmida e a massa seca das espécies.

$$
U \%=\frac{M U-M S}{M S} \times 100
$$

Sendo:

$U \%=$ teor de umidade do material combustível (\%); $M U$ = massa úmida do combustível no momento da coleta (g); e $M S=$ massa seca do material combustível (g).

Para realizar as queimas experimentais, de cada espécie foram separadas amostras e com elas, foram realizadas 50 repetições de queima de $1 \pm 0,1 \mathrm{~g}$. As queimas foram realizadas com o uso de epirradiador (Figura 1), que consiste num dispositivo elétrico, que possui um disco de $10 \mathrm{~cm}$ de diâmetro e uma chama piloto localizada a $4 \mathrm{~cm}$ de altura (GANTEAUME et al., 2013).
FIGURA 1: Epirradiador.

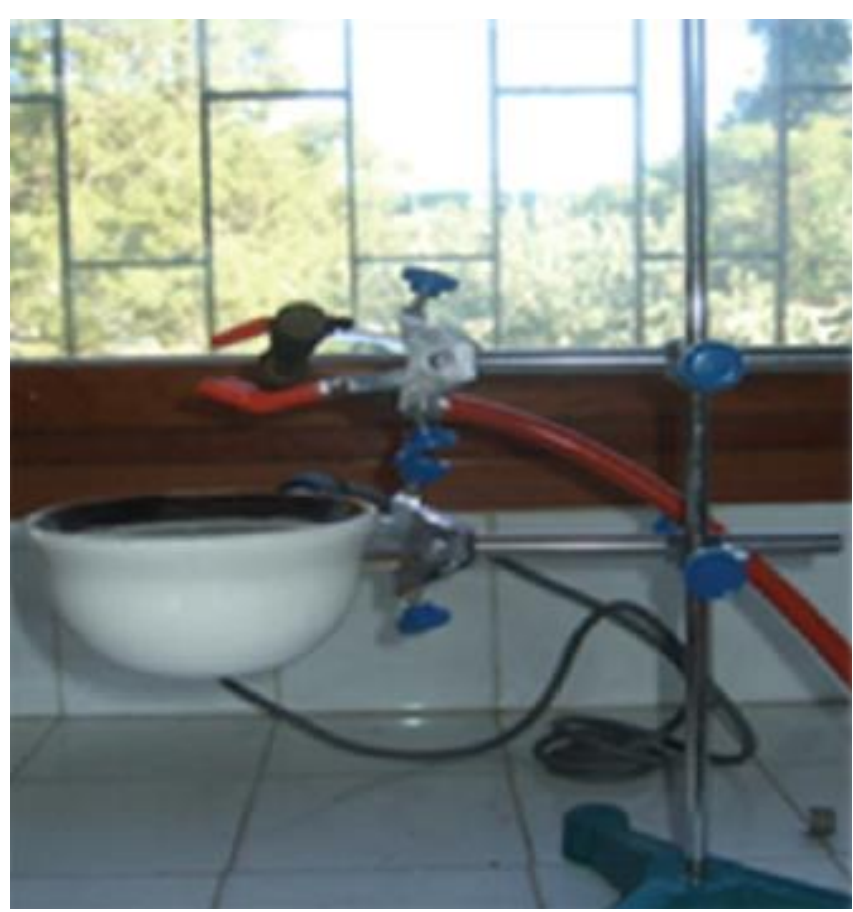

Fonte: Hachmi et al. (2011).

Kovalsyki et al. (2016) informam que, ao se manipular este equipamento, é importante que o ambiente apresente boas condições, estando livre de corrente de ar. Sendo assim, o local escolhido para o experimento foi o Laboratório do Centro de Monitoramento Ambiental e Manejo do Fogo - CEMAF, pois o ambiente apresenta as condições ideais, garantindo o bom funcionamento do epirradiador, como também mantendo acesa a chama piloto.

Ao realizar as queimas experimentais, foi adotada a metodologia descrita por Petriccione (2006), com o epirradiador, em uma faixa de temperatura controlada em torno de $600^{\circ} \mathrm{C}$, que foi a temperatura mensurada pela câmera termal no momento das queimas, a mesma temperatura utilizada por Santos et al. (2018), para avaliar a inflamabilidade de espécies do Cerrado, na região do Jalapão.

De cada amostra, foram considerados os seguintes parâmetros de inflamabilidade:

- Tempo para Ignição (TI): tempo que o material leva para iniciar a combustão;

- Duração da Combustão (DC): tempo que a chama se mantém acesa; 
- Altura de Chama (HC): altura máxima que a chama atinge no momento da queima.

Após realizar as 50 repetições de cada espécie, os dados de TI, DC e HC foram submetidos à análise estatística de comparação de médias por meio do teste Scott- Knott ao nível de 5\%. Posteriormente, foi possível obter os parâmetros de:

- Frequência de Ignição (FI): é a quantidade de vezes em que ocorreu o TI com mais frequência, considerando um TI máximo de $60 \mathrm{~s}$; queimas que ultrapassaram esse tempo foram classificadas como "queimas negativas";

- Índice de combustão (IC): se refere à intensidade de combustão de cada espécie, sendo obtida através do comprimento máximo da chama (Tabela 1);

- Valor de Inflamabilidade (VI): é atribuída de acordo com a FI e o TI (Tabela 2).
TABELA 1: Índices do valor de combustão.

\begin{tabular}{ccc}
$\begin{array}{c}\text { Índice de } \\
\text { combustão (IC) }\end{array}$ & $\begin{array}{c}\text { Designação } \\
\text { do IC }\end{array}$ & $\begin{array}{c}\text { Comprimento } \\
\text { da chama (cm) }\end{array}$ \\
\hline IC1 & Muito baixa & $<1$ \\
IC2 & Baixa & 1 a 3 \\
IC3 & Média & 4 a 7 \\
IC4 & Alta & 8 a 12 \\
IC5 & Muito alta & $>12$ \\
\hline
\end{tabular}

Fonte: Petriccione (2006).

\section{Resultados}

O teor de umidade e os valores médios das variáveis de inflamabilidade das espécies analisadas estão apresentados na Tabela 3.

TABELA 2: Índices do valor de inflamabilidade.

\begin{tabular}{ccccccc}
\hline TI & \multicolumn{7}{c}{ FI } \\
\cline { 2 - 7 } & $<\mathbf{2 5}$ & $\mathbf{2 5 - 3 8}$ & $\mathbf{3 9 - 4 1}$ & $\mathbf{4 2 - 4 4}$ & $\mathbf{4 5 - 4 7}$ & $\mathbf{4 8 - 5 0}$ \\
\hline$>32,5$ & 0 & 0 & 0 & 1 & 1 & 2 \\
$27,6-32,5$ & 0 & 0 & 1 & 1 & 2 & 2 \\
$22,6-27,5$ & 0 & 0 & 1 & 2 & 2 & 2 \\
$17,5-22,5$ & 1 & 1 & 2 & 2 & 3 & 3 \\
$12,6-17,5$ & 1 & 1 & 2 & 3 & 3 & 4 \\
$<12,6$ & 1 & 2 & 3 & 3 & 4 & 5 \\
\hline
\end{tabular}

FI - Frequência de Ignição e TI - tempo para Ignição. O valor de Inflamabilidade (VI) será classificado em função do número correspondente, em que: VI = 0 (fracamente inflamável); VI = 1 (pouco inflamável); VI = 2 (moderadamente inflamável); VI = 3 (inflamável); VI = 4 (altamente inflamável) ou VI = 5 (extremamente inflamável). Fonte: Valette (1992).

TABELA 3: Valores médios das variáveis de inflamabilidade para as espécies arbóreas em áreas do Cerrado.

\begin{tabular}{lccc}
\hline \multicolumn{1}{c}{ Espécies } & TI & DC & HC \\
\hline Lafoensia pacari & $3,42^{\mathrm{A}}$ & $0,59^{\mathrm{A}}$ & $19,18^{\mathrm{D}}$ \\
Terminalia argentea Mart. & $4,21^{\mathrm{A}}$ & $2,00^{\mathrm{A}}$ & $14,70^{\mathrm{B}}$ \\
Acacia polyphylla & $4,49^{\mathrm{A}}$ & $2,55^{\mathrm{A}}$ & $13,68^{\mathrm{A}}$ \\
Byrsonima verbascifolia (L.) DC. & $5,13^{\mathrm{A}}$ & $1,94^{\mathrm{A}}$ & $20,12^{\mathrm{E}}$ \\
Caryocar brasiliense Cambess. & $9,49^{\mathrm{B}}$ & $7,76^{\mathrm{B}}$ & $15,86^{\mathrm{C}}$ \\
Protium heptaphyllum & $13,30^{\mathrm{C}}$ & $7,21^{\mathrm{B}}$ & $21,16^{\mathrm{F}}$ \\
\hline Média Geral & 6,67 & 3,67 & 17,45 \\
\hline CV (\%) & 122,39 & 118,93 & 6,02 \\
\hline
\end{tabular}

Letras iguais indicam que, ao nível de 5\%, não há diferença significativa entre as médias pelo teste Scott-Knott. TI - Tempo para Ignição (s); DC - Duração da Combustão (s); HC - Altura da Chama (cm). 
A espécie $L$. pacari apresentou o menor TI $(3,42 \mathrm{~s})$, seguida de $T$. argentea $(4,21 \mathrm{~s})$, A. polyphylla $(4,49 \mathrm{~s}), B$. verbascifolia $(5,13 \mathrm{~s})$, não havendo diferença estatística significativa entre elas. Já as espécies C. brasiliense $(9,49 \mathrm{~s})$ e $P$. heptaphyllum $(13,30 \mathrm{~s})$ apresentaram valores superiores, com diferença estatística significativa entre elas e as demais.

Em relação à $\mathrm{HC}$, nota-se uma diferença significativa entre todas as espécies. A espécie A. polyphylla $(13,68$ $\mathrm{cm})$ apresentou a menor medida, enquanto a espécie $P$. heptaphyllum $(21,16 \mathrm{~cm})$ apresentou a maior $\mathrm{HC}$.

No que se refere à duração das chamas, a menor duração ocorreu com a espécie $L$. pacari $(0,59 \mathrm{~s})$, enquanto a maior duração ocorreu com a espécie $C$. brasiliense $(7,76 \mathrm{~s})$.

Em relação ao teor de umidade, a espécie $P$. heptaphyllum, apresentou o menor valor $(99,08 \%)$, no entanto, de acordo com a Figura 2, a espécie foi considerada fracamente inflamável. Já a espécie $B$. verbascifolia, apresentou o maior teor de umidade $(224,88 \%)$, mesmo sendo considerada como uma espécie inflamável. As espécies L. pacari, T. argentea, A. polyphylla e C. brasiliense apresentaram teor de umidade $180,95 \%, 144,98 \%, 102,01 \%$ e $142,40 \%$ respectivamente.

Na Figura 2, pode-se observar os resultados de IC como também o VI de todas as espécies. Com a análise do TI, verificou-se que as espécies $C$. brasiliense, $B$. verbascifolia, $A$. polyphylla e $T$. argentea são inflamáveis. Já a espécie $L$. pacari foi considerada altamente inflamável, enquanto a espécie $P$. heptaphyllum, como já citado anteriormente, foi considerada pouco inflamável.

A HC de todas as espécies ultrapassou a medida de $12 \mathrm{~cm}$, dessa forma, todas as espécies possuem IC muito alto.

Percebe-se uma forte correlação entre os parâmetros TI e DC, TI e VI e entre DC e VI, como pode ser visto na Tabela 4.

FIGURA 2: Valor de Inflamabilidade (VI) e Índice de Combustão (IC).

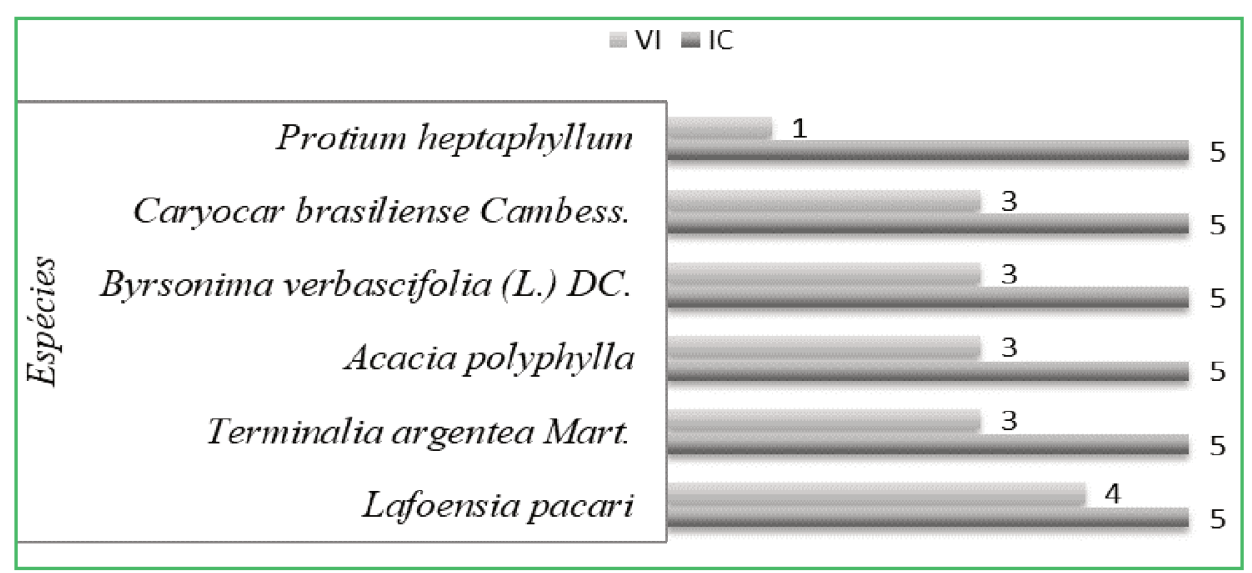

IC = 5: Índice de Combustão muito alto; O valor de inflamabilidade (VI) está classificado em função do número correspondente, em que: $\mathrm{VI}=0$ (fracamente inflamável); VI $=1$ (pouco inflamável); VI $=2$ (moderadamente inflamável); VI $=3$ (inflamável); VI $=4$ (altamente inflamável) ou VI = 5 (extremadamente inflamável). Fonte: Valette (1992).

TABELA 4: Correlação de Pearson.

\begin{tabular}{ccccc}
\hline Parâmetros & TI & DC & HC & U \\
\hline DC & $\mathbf{0 , 9 2}$ & & & \\
HC & 0,42 & 0,12 & & \\
U & $-0,49$ & $-0,51$ & 0,36 & 0,56 \\
VI & $\mathbf{- 0 , 8 6}$ & $\mathbf{- 0 , 6 8}$ & $-0,37$ & \\
\hline
\end{tabular}

Resultados obtidos a partir da fórmula de correlação de Pearson, onde se desconsiderando o sinal, coeficientes que se aproximam de $0=$ correlação fraca; e para coeficientes que se aproximam de $1=$ correlação forte. 


\section{Discussão}

A espécie $L$. pacari foi considerada altamente inflamável, no entanto, foi uma das espécies que apresentou um dos mais altos teores de umidade $(180,95 \%)$. Já a espécie $P$. heptaphyllum é a que apresentou o menor teor de umidade, maior $\mathrm{HC}$ e uma das maiores DC, sendo essa espécie considerada fracamente inflamável. De acordo com Petriccione (2006), é esperado que espécies com baixo teor de umidade sejam inflamáveis. Hachmi et al. (2011) trazem resultados que satisfazem a afirmação de Petriccione (2006), sustentando que a umidade se faz de grande importância da vegetação na ignição e na propagação do fogo. Batista et al. (2012), ao avaliarem a inflamabilidade de árvores e arbustos para a implementação de barreiras verdes no Sul do Brasil obtiveram resultados, segundo os quais não houve correlação entre umidade e inflamabilidade das espécies estudadas.

Para tanto, Batista et al. (2012) aconselham que se faz necessário estudos mais detalhados de aspectos relevantes que se associam à inflamabilidade, como, por exemplo, a presença de óleos, resinas e gomas vegetais.

Analisando a relação entre o teor de umidade e a inflamabilidade de espécies do Cerrado, Santos et al. (2018) classificaram a espécie Byrsonima verbascifolia como altamente inflamável (VI $=4)$, com a espécie estando a nível de umidade em seu estado natural. No presente estudo, a espécie foi classificada como inflamável (VI = 3) e apresentou o maior teor de umidade entre as espécies analisadas, como também uma das maiores HC. Em estudos de combustíveis florestais marroquinos, Hachmi et al. (2011) afirmaram que a HC tem influência na inflamabilidade, visto que a altura poderia ser um indicador da volatilidade de óleos contidos nas folhas. Além disso, os autores alegaram que a DC caracteriza a capacidade do combustível em sustentar a queima por um longo período de tempo.

No presente estudo, houve correlação entre o TI e o VI. A espécie Protium Heptaphyllum, classificada com pouca inflamabilidade, apresentou o maior TI, o que corrobora o que dizem Kovalsyki et al. (2016), que, para a espécie ser considerada útil em cortinas de segurança, é esperado que os valores de TI sejam altos.
Após análise dos resultados obtidos, pode-se perceber que não houve correlação entre teor de umidade e inflamabilidade das espécies, entretanto, houve correlação entre o TI, DC e o VI. Tais resultados confirmam Hernando (2009), ao dizer que o TI é uma das principais variáveis relacionadas à inflamabilidade.

Conclui-se que, das espécies estudadas, quatro foram classificadas como inflamáveis (Caryocar brasiliense Cambess., Byrsonima verbascifolia (L.) DC., Acacia polyphylla e Terminalia argentea Mart) e uma foi classificada como altamente inflamável (Lafoensia pacari.), não sendo recomendado o uso destas em cortinas de segurança. Por outro lado, a espécie Protium Heptaphyllum apresenta potencial para uso em cortinas de segurança, por ter sido classificada como pouco inflamável $(\mathrm{VI}=1)$.

É importante a realização de mais experimentos, em que se possa analisar, por exemplo, a presença dos compostos químicos, como também a estruturas das plantas, na influência da inflamabilidade das espécies florestais.

\section{Referências}

ANDERSON, H. E. Forest fuel ignitibility. Fire Technology, London, v. 6, n. 4, p. 312-319, 1970.

BATISTA, A. C. Incêndios florestais. Recife: Imprensa Universitária da UFRPE, 1990. 115 p.

BATISTA, A. C.; BIONDI, D.; TETTO, A. F.; ASSUNÇÃO, R. de; TRES, A.; TRAVENISK, R. C. C.; KOVALSYKI, B. Evaluación de la inflamabilidad de árboles y arbustos utilizados en la implementación de barreras verdes en el sur del Brasil. In: SYMPOSIUM ON FIRE ECONOMICS, PLANNING, AND POLICY: CLIMATE CHANGE AND WILDFIRES, 4, 2012, Albany. Proccedings... Albany: U.S. Department of Agriculture, Forest Service, Pacific Southwest Research Station, 2012. p. 256264.

BATISTA, E. K. L.; RUSSELL-SMITH, J.; FRANÇA, H.; FIGUEIRA, J. E. C. An evaluation of contemporary savanna fire regimes in the Canastra National Park, Brazil: Outcomes of fire suppression policies. Journal of Environmental Management, New York, v. 205, p. 40-49, 2018.

ESSAGHI, S.; HACHMI, M.; YESSEF, M.; DEHHAOUI, M.; EL AMARTY, F. Assessment of flammability of Moroccan forest fuels: new approach to estimate the Flammability Index. Forests, Basel, v. 8, n. 11, p. 1-16, 2017.

ETLINGER, M. G.; BEALL, F. C. Development of a laboratory protocol for fire performance of landscape plants. International Journal of Wildland Fire, Victoria, v. 13, p. 479-488, 2004. 
FRÉJAVILLE, T.; CURT, T.; CARCAILLET, C. Higher potential fire intensity at the dry range margins of European mountain trees. Journal of Biogeography, New York, v. 45, n. 9, p. 2003-2015, 2018.

HACHMI, M'H.; SESBOU, A.; BENJELlOUN, H.; El HANDOUZ, N.; BOUANANE, F. A Simple Technique to Estimate the Flammability Index of Moroccan Forest Fuels. Journal of Combustion, London, v. 2011, p. 1-11, 2011.

HERNANDO, C. L. Combustibles forestales: inflamabilidad. In: VÉLEZ, R. M. (Coord.). La defensa contra incêndios forestales: fundamentos y experiências, 2. ed. Madrid: McGraw-Hill, 2009. p. 3-6.

GANTEAUME, A.; JAPPIOT, M.; LAMPIN, C.; GUIJARRO, M.; HERNANDO, C. Flammability of some ornamental species in wildland-urban interfaces in Southeastern France: laboratory assessment at particle level. Journal of Environmental Management, New York, v. 52, n. 2, p. 467-480, 2013.

GANTEAUME, A.; MARIELLE, J.; CORINNE, L.; THOMAS, C.; LAURENT, B. Effects of vegetation type and fire regime on flammability of undisturbed litter in Southeastern France. Forest Ecology and Management, Amsterdam, v. 261, n. 12, p. 22232231, 2011.

KOVALSYKI, B; CASIMIRO JUNIOR, L. D.; ASSUNÇÃO, R.; TRAVENISK, R. C. C.; TETTO, A. F.; BATISTA, A. C. Avaliação de espécies arbóreas para composição de cortinas de segurança contra incêndios florestais. Nativa, Sinop, v. 7, n. 2, p. 197-203, 2019.

KOVALSYKI, B.; TAKASHINA, I. K.; TRES, A.; TETTO, A. F.; BATISTA, A. C. Inflamabilidade de espécies arbóreas para uso em cortinas de segurança na prevenção de incêndios florestais. Pesquisa Florestal Brasileira, Colombo, v. 36, n. 88, p. 387-393, 2016.

MCLAUCHLAN, K. K.; HIGUERA, P. E.; MIESEL, J. ROGERS, B. M.; SCHWEITZER, J.; SHUMAN, J. K. et al. Fire as a fundamental ecological process: Research advances and frontiers. Journal of Ecology, London, v. 108, n. 5, p. 2047-2069, 2020.
MOLINA, J. R.; MARTÍN, T.; SILVA, F. R.; HERRERA, M. Á. The ignition index based on flammability of vegetation improves planning in the wildland-urban interface: a case study in southern spain. Landscape and Urban Planning, San Diego, v. 158, p. 129138, 2017.

NELSON, R. M. Water relations of forest fuels. In: JOHNSON, E. A.; MIYANISHI, K. (Ed.). Forest fires: behavior and ecological effects. Chapter 4. London: Academic Press, 2001. p. 79-150.

PETRICCIONE, M. Infiammabilità della lettiera di diverse specie vegetali di ambiente Mediterraneo. 2006. $48 \mathrm{f}$. Tese (Doutorado em Biologia Aplicada) - Università Degli Studi Di Napoli Federico II, Napoli. 2006.

SANTOS, M. M.; BATISTA, A. C.; CARVALHO, E. V.; SILVA, F. C.; PEDRO, C. M.; GIONGO, M. Relationships between moisture content and flammability of campestral Cerrado species in Jalapão. Revista Brasileira de Ciências Agrárias, Recife, v. 13, n. 4, p. 1-9, 2018.

SILVEIRA, E. M. O.; TERRA, M. C. N. S.; STEEGE, H.; MAEDA, E. E.; ACERBI JÚNIOR, F. W.; SCOLFORO, J. R. S. Carbondiversity hotspots and their owners in Brazilian southeastern Savanna, Atlantic Forest and Semi-Arid Woodland domains. Forest, Ecology and Management, Flagstaff, v. 452, 117575, 2019.

SIMPSON, K. J.; RIPLEY, B. S.; CHRISTIN, P.; BELCHER, C. M.; LEHMANN, C. E. R.; THOMAS, G. H.; OSBORNE, C. P. Determinants of flammability in savanna grass species. Journal of Ecology, London, v. 104, n. 1, p. 138-148, 2015.

SOARES, R. V.; BATISTA, A. C. Incêndios florestais: controle, efeitos e uso do fogo. Curitiba: Ronaldo Viana Soares, 2007. 264 p.

VALETTE, J. C. Inflammabilities of mediterranean species. Porto Carras: Research and Development of the European Commission European School of Climatology and Natural Hazards, 1992. $12 \mathrm{p}$. 